\title{
Spectral-Amplitude-Coded OCDMA Optimized for a Realistic FBG Frequency Response
}

\author{
Julien Penon, Student Member, IEEE, Ziad A. El-Sahn, Student Member, IEEE, \\ Leslie A. Rusch, Senior Member, IEEE, and Sophie LaRochelle, Member, IEEE
}

\begin{abstract}
We develop a methodology for numerical optimization of fiber Bragg grating frequency response to maximize the achievable capacity of a spectral-amplitude-coded optical code-division multiple-access (SAC-OCDMA) system. The optimal encoders are realized, and we experimentally demonstrate an incoherent SAC-OCDMA system with seven simultaneous users. We report a bit error rate (BER) of $2.7 \times 10^{-8}$ at $622 \mathrm{Mb} / \mathrm{s}$ for a fully loaded network (seven users) using a 9.6-nm optical band. We achieve error-free transmission $\left(B E R<1 \times 10^{-9}\right)$ for up to five simultaneous users.
\end{abstract}

Index Terms-Fiber Bragg gratings (FBGs), frequency encoded (FE) optical code-division multiple access (OCDMA), frequency encoding, intensity noise, spectral-amplitude-coded (SAC) OCDMA.

\section{INTRODUCTION}

A VARIETY of technologies and architectures are currently under study for optical code-division multipleaccess (OCDMA), targeting different applications, bit rates, and throughputs. In this paper, we focus on spectral amplitude coding for access networks due to the potential for low-cost client premises equipment. Access networks deliver services such as video on demand, multichannel high-definition television (HDTV), ultrahigh-speed Internet, etc., to residential customers. This type of traffic is known to be bursty, and code-division multiple-access (CDMA) in the electrical domain has been demonstrated to be very efficient in this scenario as compared to carrier-sense multiple-access networks (Ethernet, ALOHA, etc.) [1]. The main challenge that faces researchers is the efficient transfer of this advantage to the optical domain, with many methods under investigation [2]-[6]. Some OCDMA systems achieve high bit rate but are quite complex and expensive because they use mode-locked lasers, nonlinear thresholders, etc. [7]. These components are of high cost and not easily available commercially. Achieving spectralamplitude-coded (SAC) encoding/decoding by means of bulk optics (diffraction gratings, mirrors, amplitude masks, etc.) has high losses (11-13 dB), and such setups are difficult to adjust and to stabilize [8]. This paper focuses on low-cost

Manuscript received August 25, 2006; revised February 2, 2007.

The authors are with the Center for Optics, Photonics, and Lasers, Department of Electrical and Computer Engineering, Université Laval, Sainte-Foy, QC G1K 7P4, Canada (e-mail: jpenon@gel.ulaval.ca; ziad.elsahn.1@ulaval.ca; rusch@gel.ulaval.ca; larochel@gel.ulaval.ca).

Color versions of one or more of the figures in this paper are available online at http://ieeexplore.iee.org.

Digital Object Identifier 10.1109/JLT.2007.893917 architectures offering moderate bit rates $(622 \mathrm{Mb} / \mathrm{s}$ to gigabit Ethernet rates) that are suitable, for example, for passive optical networks (PONs) [9]. A variety of technologies have been proposed for SAC-OCDMA encoders; however, we focus our attention on the use of fiber Bragg gratings (FBGs) as an ideal component to achieve our system goals of stable, low-cost, and low-loss encoders.

An incoherent SAC-OCDMA system using superimposed FBGs in reflection as encoders/decoders was tested in [10] for a system that uses m-sequence codes. The authors identified many points where the capacity of SAC systems could be improved and their cost could be reduced: 1) use of codes with fixed minimal cross correlation instead of m-sequences, 2) use of FBGs in transmission, and 3) minimal frequency guard bands. In this paper, we adopt their suggestions and demonstrate an improved system. In [10], a system with an optical bandwidth of $6 \mathrm{~nm}$ operating at $622 \mathrm{Mb} / \mathrm{s}$ showed a $10^{-4}$ bit error rate (BER) floor with only three active users. At an optical bandwidth of $9.6 \mathrm{~nm}$, we achieve seven active users with a BER floor of $2.7 \times 10^{-8}$ at $622 \mathrm{Mb} / \mathrm{s}$. In order to get this improved performance, we needed to optimize the shape of the achievable FBG spectral response (spectral shape) to best achieve the suggestion of "minimal frequency guard bands." In [10], a simulation tool was described to predict the performance of SAC-OCDMA systems as a function of the encoder/decoder spectral response using theory derived from Goodman [11]. Their encoders/decoders were written with significant frequency guard bands between frequency bins (with these bins being a partition of the available bandwidth into uniform segments for coding). The original intent of the guard bands was to reduce multiple-access interference (MAI) by assuring that when two codes do not have a given bin in common, no energy would "leak through" one bin to the other. Unfortunately, MAI was not the principal source of system impairment, and this actually reduced the system capacity. The principal source of impairment was interferometric beat noise, which leads to intensity noise, which is also known as phaseinduced intensity noise.

Ultimately, all SAC-OCDMA systems are intensity noise limited, even if they use coherent multifrequencies sources having the same center frequencies [13]. In this paper, as in [10], we focus on the use of broadband sources due to their low cost and large emission bandwidth compared to coherent sources. The intensity noise in an incoherent system is inversely proportional to the effective optical bandwidth (the occupied bandwidth and not the spanned bandwidth) and proportional to the electrical bandwidth. For a given bit rate, systems with 
greater optical bandwidth would offer better performance in terms of BER or, for fixed BER, could accommodate more users for greater capacity.

Since intensity noise is the principal noise source, frequency guard bands reduce the occupied bandwidth; hence, they reduce the effective optical bandwidth and, therefore, the capacity. If ideal rectangular filters could be achieved, the encoders would carve out truly orthogonal frequency bins while exploiting all available bandwidth. Realistic FBGs that can be written in a cost-effective manner will have finite rolloff, leading to a tradeoff between MAI and intensity noise. In this paper, we use the simulator proposed in [10] as a design tool to numerically trade off the MAI versus intensity noise. This tradeoff requires the identification of further constraints on the code family to achieve optimization, as will be described later. We simulate achievable FBG spectral responses with various levels of overlap between bins. These spectral responses are then used to predict the BER floor in a SAC-OCDMA system. The optimum spectral response is determined, and a set of encoders/decoders is realized. These components are then tested in a system configuration similar to that in [12].

The remainder of this paper is organized as follows: In Section II, we describe SAC-OCDMA networks and their principles of operation, explaining the role of each component. Section III discusses the computation of the BER floor in intensity-noise-limited SAC-OCDMA networks. In Section IV, we describe our encoder optimization, including the issues of code choice and the apodization profile used for writing FBGs. In Section V, we present our experimental setup and BER measurements for the optimized system. Finally, we conclude with suggestions for further optimizations that might be attained.

\section{SAC-OCDMA SYSTEM}

Tree architectures are efficient for fiber-to-the-home or the so-called "last mile" market. A network of this type that employs only passive optical components (couplers, splitters, circulators, etc.) is referred to as PON and has low installation and management costs, as compared with active networks. The standards for today's PON have bit rates per household in the range of $20-30 \mathrm{Mb} / \mathrm{s}$, which are inadequate to meet growing consumer demand (multichannel HDTV, ultrahigh-speed Internet, video on demand, etc.). Future PONs will exploit OCDMA or wavelength-division multiplexing to increase perclient rates up to $1.25 \mathrm{~Gb} / \mathrm{s}$, with 16 or 32 simultaneous clients per network. We propose a network using SAC-OCDMA at the physical layer. Such a network assigns a unique spectral code to each user, as shown in Fig. 1. SAC-OCDMA takes a data-modulated optical signal with wide spectral content, e.g., multiple lasers, multifrequencies source, or a broadband source, and encodes each bit in the spectral domain. In this example, a low-cost light-emitting diode (LED) is directly modulated, followed by a passive encoder ENC. The encoder is an FBG working in transmission that takes a broadband source and filters out all spectral content, except those frequencies included in the user's unique spectral code. All the users in the system share the same optical bandwidth and contain frequency elements from the same band; they access the channel

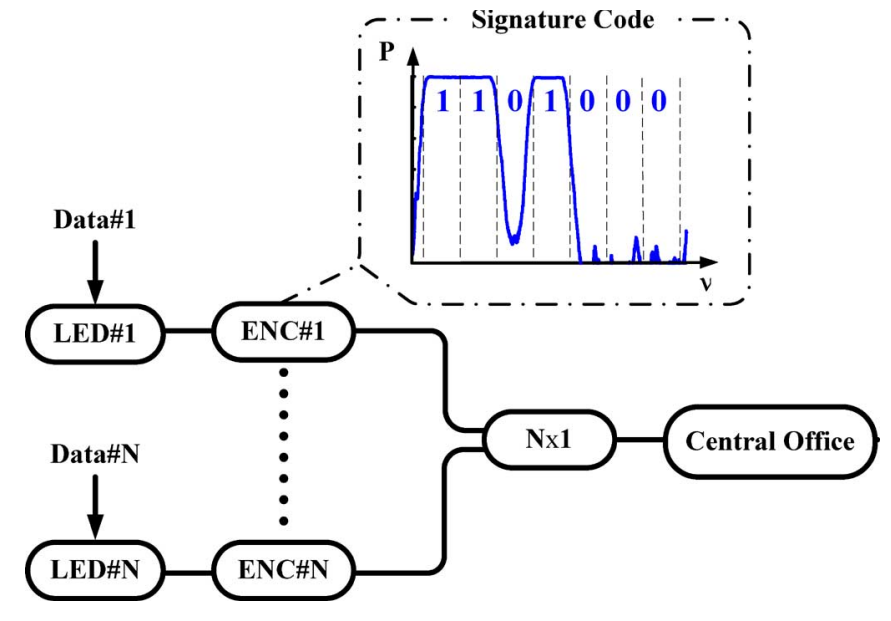

Fig. 1. SAC-OCDMA network.

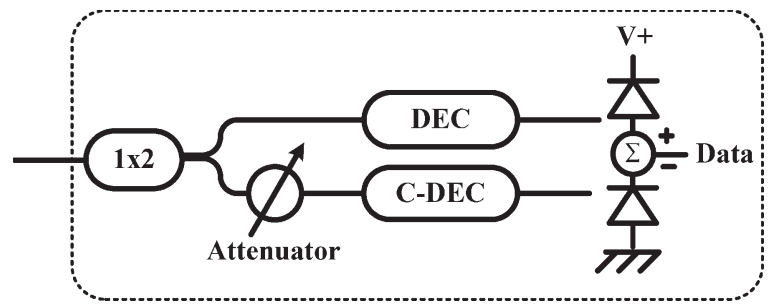

Fig. 2. Balanced detection in SAC-OCMA network.

asynchronously and without coordination. An $N \times 1$ coupler is used to combine all signals onto one fiber to the central office (CO). The receiver for SAC-OCDMA systems is the balanced detector illustrated in Fig. 2 and would be the same at the $\mathrm{CO}$ or client premises. Balanced detection [4] eliminates MAI for codes with fixed cross correlation, leaving only intensity noise. The upper arm contains an FBG with the decoder DEC identical to the ENC for the data to be received. In the lower arm, the complementary decoder C-DEC is orthogonal to the ENC spectral response, i.e., it contains only the frequency bins that are not present in the ENC. An optical attenuator is inserted to achieve balanced detection and is adjusted according to the used codes.

\section{Simulation of System Performance}

The main limiting noise at high received power is the intensity noise generated by the incoherent broadband source. The intensity noise goes as the square of the photocurrent, unlike shot noise, which varies linearly, and thermal noise, which does not depend on the photocurrent [14]. In [15], intensity noise led to a BER floor, even with only one interferer. In [10], the simulation results were presented to fit the BER floor, which was ultimately attributed to the low ratio of optical bandwidth to electrical bandwidth and high cross correlation of the used codes. In this section, the methodology of [10] is used to predict the BER floor as a function of all components' spectral responses.

Let $S_{\mathrm{ENC}}^{k}(\nu), S_{\mathrm{DEC}}(\nu)$, and $S_{\mathrm{C}-\mathrm{DEC}}(\nu)$ represent the spectral response of the $k$ th user's ENC, the DEC, and the C-DEC, respectively, where $\nu$ is the optical frequency. 
To avoid burdensome notation, the last two expressions have no superscript as they refer to a unique detection device for the desired user, which we refer to as user\#1. $S_{\text {SOURCE }}(\nu)$ represents the spectrum of the broadband source, which is taken to be identical for all users. Let $F_{\mathrm{DEC}}^{k}(\nu)$ and $F_{\mathrm{C}-\mathrm{DEC}}^{k}(\nu)$ represent the spectrum after DEC and C-DEC, respectively, when only the $k$ th user is active. They are given by

$$
\begin{aligned}
F_{\mathrm{DEC}}^{k}(\nu) & =S_{\mathrm{SOURCE}}(\nu) \cdot S_{\mathrm{ENC}}^{k}(\nu) \cdot S_{\mathrm{DEC}}(\nu) \\
F_{\mathrm{C}-\mathrm{DEC}}^{k}(\nu) & =S_{\mathrm{SOURCE}}(\nu) \cdot S_{\mathrm{ENC}}^{k}(\nu) \cdot S_{\mathrm{C}-\mathrm{DEC}}(\nu) .
\end{aligned}
$$

The spectral responses of components other than the source and the encoders are considered ideal, i.e., flat. Let $E R$ be the extinction ratio of the modulated broadband signal at the receiver and $K$ be the total number of users in the system; the number of active interferers is $q \in\{0,1, \ldots, K-1\}$. Let $d$ be a row vector of length $K$, where each element $d_{k}$ takes one of three values $(0,1,1 / \mathrm{ER})$. The $k$ th element of $\underline{d}$ is 0 if the $k$ th user is inactive, 1 if the $k$ th user is active and transmitting a logical " 1 ," and $1 / E R$ if the $k$ th user is active and transmitting a logical " 0 ." Finally, let $F_{\mathrm{DEC}}(\nu, \underline{d})$ and $F_{\mathrm{C}-\mathrm{DEC}}(\nu, \underline{d})$ be the spectrum striking the photodiodes at the decoder and the complementary decoder arms, respectively. These spectra are the sum of all active users' spectra and, thus, a function of data vector $\underline{d}$ and are given by

$$
F_{\mathrm{DEC}}(\nu, \underline{d})=\underline{d} \cdot\left[\begin{array}{c}
F_{\mathrm{DEC}}^{1}(\nu) \\
\vdots \\
F_{\mathrm{DEC}}^{k}(\nu) \\
\vdots \\
F_{\mathrm{DEC}}^{K}(\nu)
\end{array}\right]=\sum_{k=1}^{K} d_{k} \cdot F_{\mathrm{DEC}}^{k}(\nu) .
$$

A similar equation holds for $F_{\mathrm{C}-\mathrm{DEC}}(\nu, \underline{d})$. Having determined $F_{\mathrm{DEC}}(\nu, \underline{d})$ and $F_{\mathrm{C}-\mathrm{DEC}}(\nu, \underline{d}), \gamma_{\underline{d}}$, which is the signal-tonoise ratio (SNR) as a function of $\underline{d}$, can be found for each photodiode. The analysis used by Goodman [11] gives the following expression for $\gamma_{d}$ :

$$
\gamma_{\underline{d}}=\frac{m \cdot|H(0)|^{2} \cdot\left(\int_{-\infty}^{+\infty} F_{\mathrm{DEC}}(\nu, \underline{d}) d \nu\right)^{2}}{\int_{-\infty}^{+\infty}\left(\int_{-\infty}^{+\infty} F_{\mathrm{DEC}}(\nu, \underline{d}) F_{\mathrm{DEC}}(\nu+f, \underline{d}) d \nu\right)|H(f)|^{2} d f} .
$$

A similar equation holds for the SNR of the C-DEC. The polarization is accounted for by $m(m=2$ for a nonpolarized broadband source and $m=1$ for a polarized signal); $H(f)$ is the normalized frequency response of the photoreceiver including the photodiode, electrical amplifiers and filters, where $f$ is the baseband electrical frequency.

For an electrical response with $T$ time constant, integrated intensity $W$ can be expressed as follows:

$$
W=\int_{t-T}^{T} I(\varepsilon) \cdot d \varepsilon
$$

where $I$ is the time-varying intensity. Considering the spectrum hitting the photodiode in the DEC arm, the average integrated intensity $\overline{W_{d}}$, depending on data vector $\underline{d}$, can be calculated from the following equation:

$$
\overline{W_{\underline{d}}}=\int_{-\infty}^{+\infty} F_{\mathrm{DEC}}(\nu, \underline{d}) d \nu .
$$

According to Goodman [11], the probability density function (PDF) of the integrated intensity is approximated as a gamma density parameterized by the SNR $\gamma_{d}$ and mean $\overline{W_{d}}$. On the $\mathrm{DEC}$ arm, the PDF of $W$ is given by

$$
f_{\underline{d}}^{\mathrm{DEC}}(W)=\left(\frac{\gamma_{\underline{d}}}{\overline{W_{\underline{d}}}}\right)^{\gamma_{\underline{d}}} \frac{W^{\gamma_{\underline{d}}-1} \exp \left(-W_{\underline{d}} \cdot \gamma_{\underline{d}} / \overline{W_{\underline{d}}}\right)}{\Gamma\left(\gamma_{\underline{d}}\right)}
$$

where $\Gamma$ is the standard gamma function. A similar expression applies for the PDF $f_{d}^{\mathrm{C}-\mathrm{DEC}}(W)$ on the C-DEC arm. As in [10], the spectra are assumed to be orthogonal, i.e., independent on the two arms of the balanced photodetector. We wish to calculate the BER as a function of the number of active interferers $q$. Recall that there are at most $q$ nonzero elements of $\underline{d}$. The PDF $p d f(W, q)_{d}$ at the output of the balanced detector is calculated by convolving together the PDFs at each arm [16], i.e.,

$$
p d f(W, q)_{\underline{d}}=f_{\underline{d}}^{\mathrm{DEC}}(-W) \otimes f_{\underline{d}}^{\mathrm{C}-\mathrm{DEC}}(W) .
$$

Since weak overlap between bins exists in realistic systems, the arms are not truly independent, and other considerations in calculating $p d f(W, q)_{\underline{d}}$ will be discussed in Section V-B.

In order to derive the BER floor for a certain number of active interferers $q$, the PDF is averaged over all possible data vectors $\underline{d}$; note that all possible sets of $q$ active interferers must be found. For a given code cardinality $K$, there are $(K-$ $1) ! /(K-1-q) ! q$ ! possible sets of $q$ active interferers and $2^{q}$ distinct data vectors for each set. Suppose that user\#1, which is the desired user, is transmitting a logical " 0 ;" the corresponding averaged PDF $p d f(W, q)_{d_{1}=1 / E R}$ is given as follows:

$$
p d f(W, q)_{d_{1}=1 / E R}=\frac{(K-1-q) ! q !}{(K-1) !} \frac{1}{2^{q}} \sum_{\substack{\text { all comb. } \\ \text { of } q \text { inter. }}} \sum_{\substack{\text { all data } \\ \text { vectors } \\ d_{1}=1 / E R}} p d f(W, q)_{\underline{d}} .
$$

The outer summation covers all sets of $q$ interferers (the amount of interference could vary with which particular interferers are active), and the inner summation covers all data vectors with the desired user transmitting logical " 0 ." A similar expression applies for the PDF $p d f(W, q)_{d_{1}=1}$ when user\#1 transmits a logical "1." Finally, assuming equally probable data bits, the BER floor for user\#1 can be calculated from the previously obtained results using the following expression:

$\operatorname{BER}_{\text {user } \# 1}(q)=\frac{1}{2} \int_{\eta}^{+\infty} p d f(W, q)_{d_{1}=1 / E R} d W$

$$
+\frac{1}{2} \int_{-\infty}^{\eta} p d f(W, q)_{d_{1}=1} d W
$$




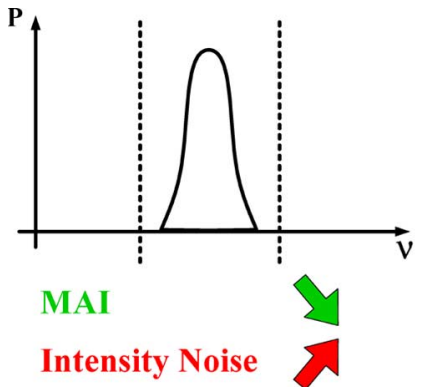

(a)

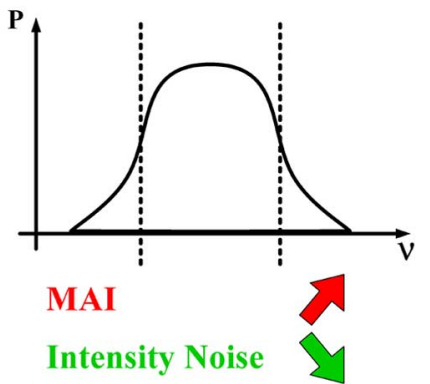

(b)

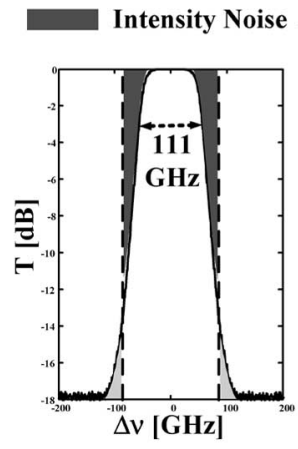

(c)

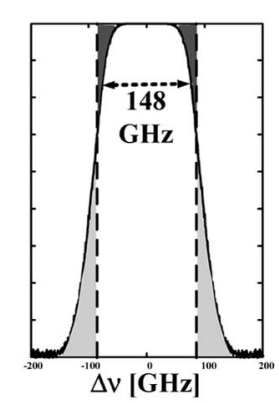

(d)
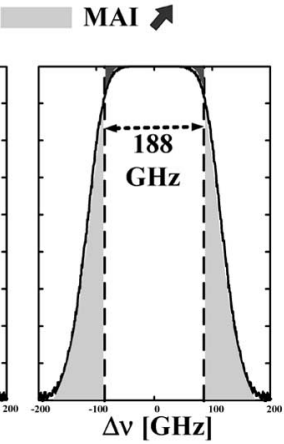

(e)
Fig. 3. Spectral response of a bin with a 3-dB bandwidth value. (a) Narrow, (b) broad, (c) $102 \mathrm{GHz}$, (d) $139 \mathrm{GHz}$, and (e) $172 \mathrm{GHz}$.

where $\eta$ is the decision threshold at the receiver. The optimal threshold can be found numerically. Calculation of the BER floor is used in the next section to optimize the encoder/decoder spectral responses and minimize the BER.

\section{Code Optimization for Realistic FBG}

In this section, we describe the methodology for the numerical optimization of the FBG spectral response. Our goal is to maximize the achievable capacity of SAC-OCDMA systems by proper selection of the code family, as well as the design of the FBG apodization profile, thus achieving minimal frequency guard bands [10]. The BER floor is then predicted by inserting the FBG spectral responses in the equations of Section III.

When an encoder design strategy leads to unequal quality of service (QoS) between users, it is difficult to find an unambiguous optimization strategy-overall network throughput, BER of a favored (higher paying) client, average BER over all clients, etc. When allowing overlapping frequency bins, we can have unequal QoS among users if the number of successive ones varies from code to code. Note that for ideal rectangular bins or widely spaced frequency bins (large guard bands) [10], the number of successive ones does not impact QoS. In order to assure roughly equal QoS among all users, we consider cyclic code families, where all but one code have the same run length of successive ones. Successive ones are attractive for overlapping, as the effective optical bandwidth is increased since adjacent overlapping bins become essentially one wide bin.

We consider the two principal noise sources in incoherent SAC-OCDMA: the intensity noise, which is inversely propor-
TABLE I

$\operatorname{BIBD}$ CODES UsED $(L=7, w=3, \lambda=1)$

\begin{tabular}{ccc}
\hline User & ENC or DEC & C-DEC \\
\hline$\# 1$ & 1101000 & 0010111 \\
$\# 2$ & 0110100 & 1001011 \\
$\# 3$ & 0011010 & 1100101 \\
$\# 4$ & 0001101 & 1110010 \\
$\# 5$ & 1000110 & 0111001 \\
$\# 6$ & 0100011 & 1011100 \\
$\# 7$ & 1010001 & 0101110 \\
\hline \hline
\end{tabular}

tional to the effective optical bandwidth and the MAI. Previous systems [10] used significant guard bands, as in Fig. 3(a), that led to greater intensity noise. As shown in Fig. 3(b), allowing overlap between adjacent frequency bins increases the effective optical bandwidth and thus reduces the intensity noise. However, the orthogonality of the two adjacent bins is lost, leading to higher MAI. Clearly, a tradeoff exists between MAI and intensity noise. Different overlap configurations must be tested in order to obtain optimum performance.

\section{A. Code Constraints}

Code choice is a major issue in SAC-OCDMA systems due to the significant impact on global performance. Many codes were proposed by several research groups [17]-[20], but they usually consider long code length (hundreds of frequency bins) and a square frequency response, which is impossible to achieve experimentally. This last assumption leads to the zeroing out of MAI after balanced detection when using constant crosscorrelation codes. In [10], Ayotte et al. used m-sequence codes, leading to severe intensity noise that limited system performance. They reported that the high cross correlation between codes was the major reason for BER floors, even with only two active users in their system. To solve this issue, they recommended the use of unity cross-correlation codes such as modified quadratic congruence (MQC) codes [21].

In this paper, balanced incomplete block design (BIBD) codes [22] are proposed because of their unity cross correlation and their cyclic property. The cardinality of BIBD codes, which equals the code length, is also higher than that of MQC codes. BIBD codes exist for any prime power, so that more code length choices are possible than with MQC codes. Codes having length $L=7$, weight $w=3$, and cross correlation $\lambda=1$ were selected to match equipment availability. This choice allows a proof of concept, but systems supporting more users are just as easily achievable with proper equipment. The codes used are illustrated in Table I. The ENCs and DECs have identical codes, whereas the C-DECs consist of the complementary code of their corresponding ENCs. Note that all codes contain one run of two consecutive ones, except for user\#7, in order to achieve roughly equal QoS. While cyclic codes are not required, they have the property of preserving the length and number of runs of logical ones, except when the runs are split by a cyclic shift from end of line to front of line. 


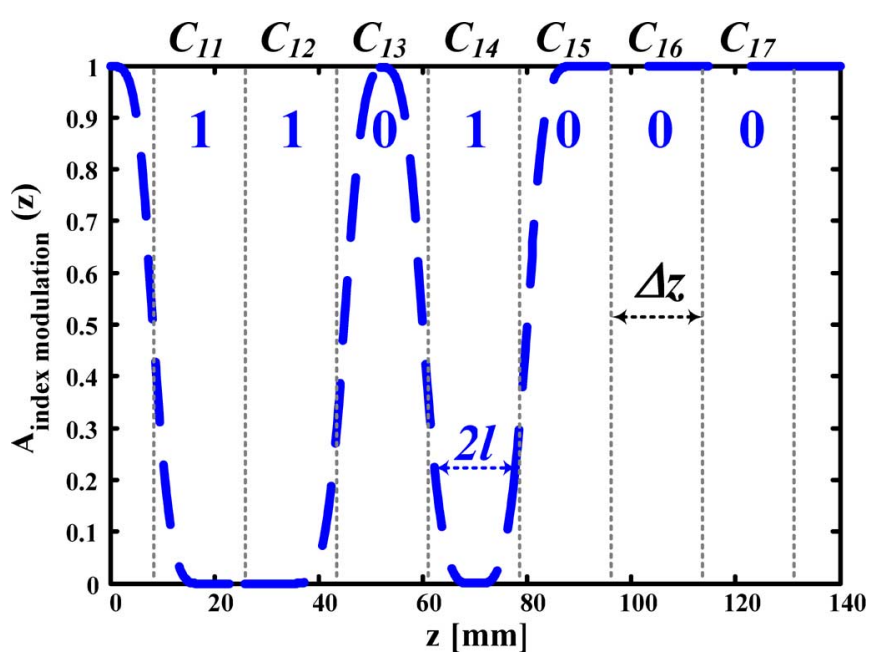

Fig. 4. Apodization profile $A^{1}(z)$ of ENC\#1 with $l=8.5 \mathrm{~mm}, \Delta z=$ $17.5 \mathrm{~mm}$, and $s=2$.

\section{B. FBG Encoders Optimization}

In the experimental setup, encoding/decoding process is achieved by FBGs that are working in transmission. The apodization profile $A(z)$ of an FBG is the modulation index envelope that will be written in the fiber. The spectral response of a highly chirped FBG is simply an inverse translation of the grating apodization profile along the $z$-axis of the fiber [23]. Basically, no modulation index $(A=0)$ leads to a transmission bin, whereas a modulation index $(A=1)$ leads to a nontransmission bin, i.e., a reflective bin. An apodization profile based on super-Gaussian lobes is used in order to minimize the ripples in spectral response that cause MAI. Therefore, the apodization profile used for the $i$ th encoder along the $z$-axis of the fiber is given by

$$
A^{i}(z)=1-\sum_{j=1}^{L=7} C_{i j} \cdot \exp \left(-0.5\left(\frac{z-z_{j}}{l}\right)^{2 s}\right)
$$

where $C_{i j}=1$ (occupied bin) if the code of the $i$ th user contains frequency $j$, and $C_{i j}=0$ (unoccupied bin) elsewhere. $s$ is the super-Gaussian lobe order, and $l$ is the super-Gaussian lobe width. A linear chirped phase mask of $0.498 \mathrm{~nm} / \mathrm{cm}$ and with a length of $140 \mathrm{~mm}$ was used. Phase-mask properties impose a total optical bandwidth of $9.6 \mathrm{~nm}$ or $1200 \mathrm{GHz}$. The distance between two apodization bins is $\Delta z$, as illustrated in Fig. 4. We set $\Delta z$ to $17.5 \mathrm{~mm}$, and $s$ is fixed at $2 . l$ is the free parameter to be optimized. Fig. 4 shows an example of an apodization profile with $l=8.5 \mathrm{~mm}$. The simulated resulting transmission spectral response (dotted line) is shown in Fig. 5. This spectral response corresponds to the first encoder (ENC\#1). The experimental spectral response (solid line) is also plotted. The correspondence between the two curves is excellent. In this particular case, bin \#1 and bin \#2 are merged to form a unique transmission bin, whereas bin \#4 corresponds to the isolated transmission bin of ENC\#1.

The next step is to analyze the effect of 3-dB bin bandwidth on BER performance by varying $l$. A bit rate of $622 \mathrm{Mb} / \mathrm{s}$ is considered. For the optimization, the $E R$ is assumed to be infinite.

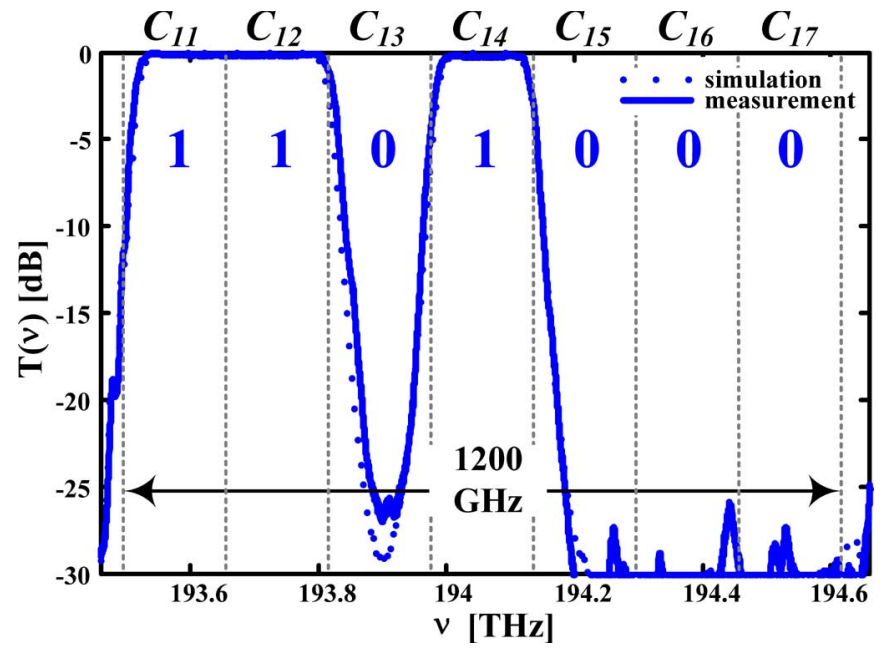

Fig. 5. Spectral response of ENC\#1 in transmission with the apodization profile of Fig. 4.

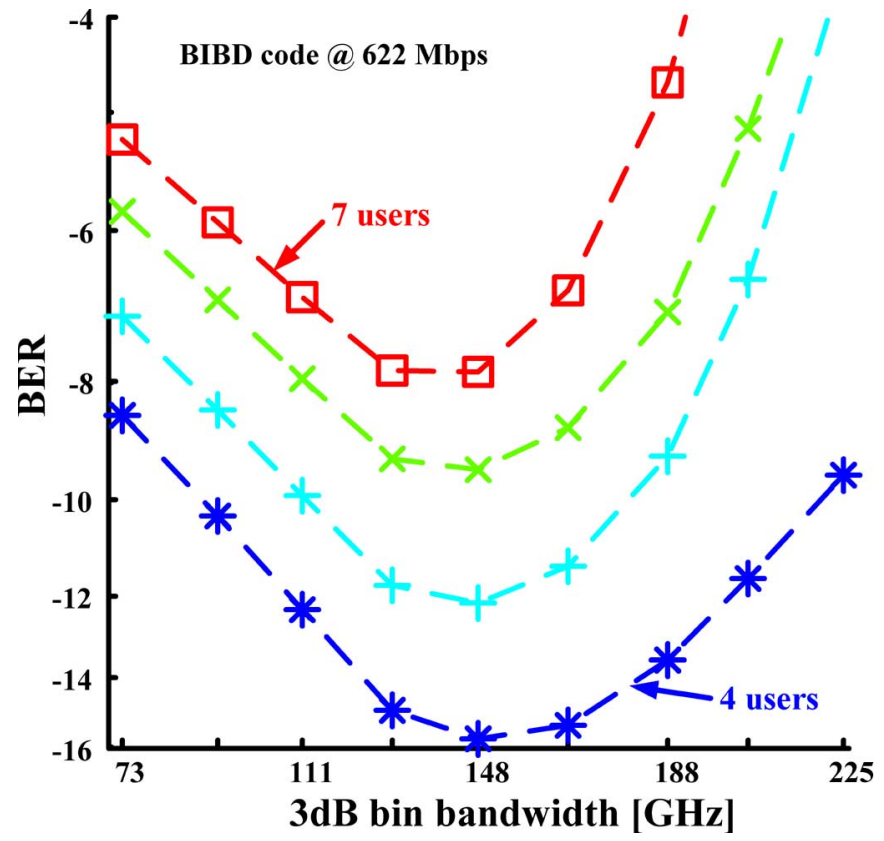

Fig. 6. BER floor versus 3-dB bin bandwidth for four to seven users.

In the simulations, all ENCs and DECs are identical. The computed BER versus 3-dB bin bandwidth is shown in Fig. 6 for different numbers of active users. As the 3-dB bin bandwidth goes from very narrow ( $73 \mathrm{GHz}$ corresponding to $l=4.5 \mathrm{~mm}$ ) to wider, the BER decreases, reaches a minimum, and then increases. Best performance occurs at a 3-dB bin bandwidth of $148 \mathrm{GHz}$ corresponding to $l=8.5 \mathrm{~mm}$ for any number of active users. We note that optimal performance occurs for $\Delta z / l \approx 2$. The reason for the BER increasing after the minimum is that the SNR improvement due to the use of wider optical bandwidth is counteracted by an increased crosstalk/overlapping between adjacent frequency bins that yield MAI. Fig. 3 presents the computed spectral responses of a single bin for different 3-dB bandwidth values [Fig. 3(c)-(e) are for 111, 148, and $188 \mathrm{GHz}$, respectively]. The configuration of Fig. 3(c) leads to high intensity noise level and low MAI, whereas the configuration of Fig. 3(e) gives a lower intensity noise level but higher MAI 


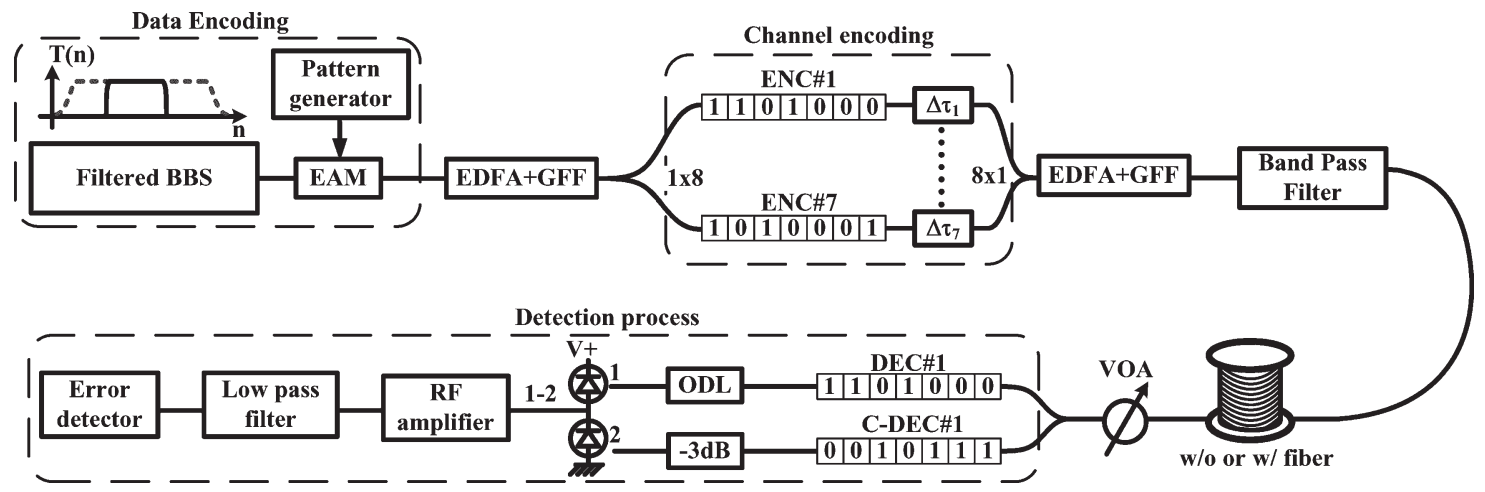

Fig. 7. Experimental setup (BBS: broadband source, EAM: electroabsorption modulator, EDFA: erbium-doped fiber amplifier, GFF: gain flattening filter, VOA: variable optical attenuator, ODL: optical delay line).

due to strong overlapping. The configuration of Fig. 3(d) leads to the best BER and corresponds to the tradeoff value that was found in Fig. 6.

The optimal FBG apodization profile $(148 \mathrm{GHz}$ for $l=$ $8.5 \mathrm{~mm}$ ) for ENC\#1 is presented in Fig. 4. The measured spectral response of ENC\#1 (solid line) is presented in Fig. 5. Isolated bin \#4 and bin \#1 and bin \#2, which were merged, have a 3-dB bandwidth of 156 and $314 \mathrm{GHz}$, respectively. Seven encoders (one per user) were written. A set of decoders and complementary decoders was written to build a balanced detection receiver. The sidelobe rejection ratio is greater than $25 \mathrm{~dB}$ for out-of-band frequencies. Normalized insertion losses over transmission bins are $0.25 \mathrm{~dB}$. All components were written according to these specifications.

\section{EXPERIMENTAL SETUP AND RESUlTS}

\section{A. Experimental Setup}

The experimental setup is illustrated in Fig. 7. A filtered $(1200 \mathrm{GHz}$ or $9.6 \mathrm{~nm}$ ) broadband source (AFC BBS 1550) is used. This filtered source is modulated by a polarizationindependent electroabsorption modulator (CyOptics EAM10-10Gb/s) at a bit rate of $622 \mathrm{Mb} / \mathrm{s}$ using a $2^{23}-1$ nonreturn-to-zero pseudorandom binary sequence. The clock signals at both transmitter and receiver sides are obtained using an HP $8657 \mathrm{~B}$ signal generator. The data stream is provided by an HP 70841B pattern generator. An erbium-doped fiber amplifier (EDFA) associated with a gain flattening filter (Teraxion) is used to compensate for the modulator losses and the $1 \times 8$ coupler that follows. Seven of the coupler branches are connected to the FBG encoders corresponding to the codes listed in Table I. Data streams are decorrelated using fiber delay lines $\Delta \tau$ of various lengths $(0,3,6,9,12,15$, and $18 \mathrm{~m})$ on each arm during the encoding process. Three meters of fiber corresponds approximately to a delay of 9.33 bits with the bit rate used. The signals from different users are then combined onto the same fiber using an $8 \times 1$ coupler. The second EDFA, which is also associated with a gain-flattening filter (Teraxion), compensates for encoding and combining losses. A bandpass filter (1200 GHz or $9.6 \mathrm{~nm}$ of bandwidth) suppresses the out-of-band amplified spontaneous emission. An optical variable attenuator (Exfo IQ-3100) is inserted to adjust the power at the receiver and to compensate for the variations of
EDFA gain when changing the number of active users. A fiber link can be included to examine the propagation over a network link. A balanced detection scheme [4] matched to user\#1 is used. The upper arm contains DEC\#1 matched to ENC\#1. An optical delay line is also inserted to achieve equal optical lengths between the two arms, which is necessary to ensure MAI cancellation. The lower arm contains C-DEC\#1, which is the complementary of DEC\#1, and a 3-dB attenuator. The $3-\mathrm{dB}$ attenuator assures that the MAI is correctly suppressed during detection for the selected codes. This $3-\mathrm{dB}$ value is theoretical because it is based on the assumption that components have ideal square spectral responses. In practice, a variable optical attenuator is adjusted to a fixed value in order to eliminate the MAI when the six interferers are present. Each arm goes into one of the two separate inputs of an $800-\mathrm{MHz}$ balanced photodiode (New Focus 1617). The electrical signal is amplified (MITEQ Inc. 0.01-500 MHz) and then low-pass filtered by a 4th order Bessel-Thomson filter (Picosecond $467 \mathrm{MHz}$ ) to remove the out-of-band high-frequency electrical noise. Finally, the electrical signal is launched into an error detector (HP 70842B) to perform the BER measurements.

In contrast to the system in [10], the FBGs are working in transmission, thereby reducing system cost by obviating circulators. Furthermore, this approach streamlines the FBG writing process so that 1) the FBGs no longer need to be superimposed as in [15], and 2) longer codes can be achieved by writing additional FBGs sequentially.

Fig. 8 shows the power spectral density (PSD) corresponding to different situations. When only the desired user is active, the PSDs after the DEC and the C-DEC are illustrated in Fig. 8(a). The cross correlation (C-DEC arm) is not equal to zero because of overlapping between frequency bins and it is very low compared to the autocorrelation (DEC arm). When only user\#6 is active, the corresponding PSDs are shown in Fig. 8(b). Only one frequency bin is present in the DEC arm with a $0.9 \times 10^{-12}$ $\mathrm{W} / \mathrm{Hz}$ level, but two bins are present in the C-DEC arm with a $0.45 \times 10^{-12} \mathrm{~W} / \mathrm{Hz}$ level. In that case, the integrated power is identical at both arms; therefore, the interferer's signal (MAI) is suppressed. In the C-DEC arm, a low-level peak $\left(<0.2 \times 10^{-12} \mathrm{~W} / \mathrm{Hz}\right)$ is also present because of overlapping. The irregularities that can be seen in the occupied bins in Fig. 8 are due to the nonflat frequency response of components such as EDFAs, broadband source, and all FBGs. 


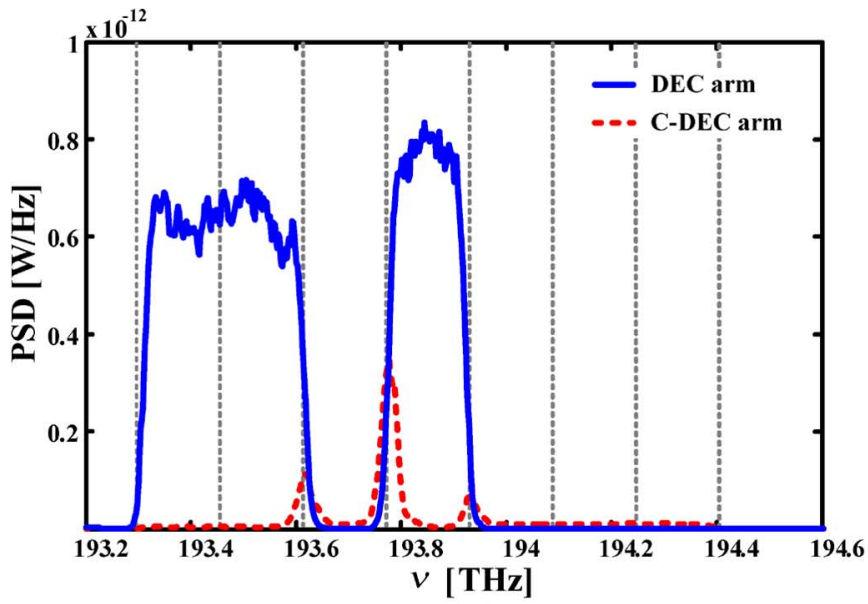

(a)

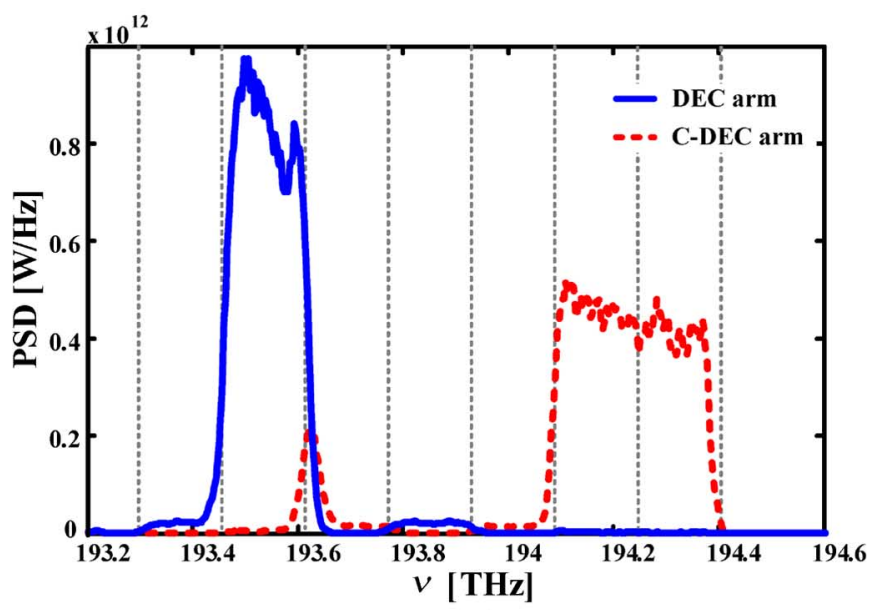

(b)

Fig. 8. PSD at the receiver side for (a) $F_{\mathrm{DEC}}^{1}(\nu)_{d=1}$ and $F_{\mathrm{C}-\mathrm{DEC}}^{1}(\nu)_{d=1}$, (b) $F_{\mathrm{DEC}}^{6}(\nu)_{d=1}$ and $F_{\mathrm{C}-\mathrm{DEC}}^{6}(\nu)_{d=1}$.

\section{B. BER Measurements}

Two configurations for BER measurements are examined: 1) without fiber and 2) with $20.522 \mathrm{~km}$ of standard SMF-28 fiber paired with a dispersion-compensating fiber (DCF) of appropriate length. All measurements are performed starting from the desired user (user\#1) to a fully loaded system (seven active users). The BER curves with no fiber (solid) and with $20.522 \mathrm{~km}$ of compensated SMF-28 (dash-dotted) are presented in Fig. 9. Error-free transmission $\left(\mathrm{BER}<1 \times 10^{-9}\right)$ can be achieved for up to five active users in the network in both configurations. Without fiber, the BER floors are reported for five to seven active users at $2.4 \times 10^{-11}, 9.5 \times 10^{-10}$, and $2.7 \times 10^{-8}$, respectively. When SMF-28+DCF fiber is used, BER floors change to $3.2 \times 10^{-11}, 1.9 \times 10^{-9}$, and $4.5 \times 10^{-8}$, respectively. BER performances are similar for both cases, showing that no significant penalty results when propagating through a $20-\mathrm{km}$ link. This proves that the chromatic dispersion can be adequately compensated at $622 \mathrm{Mb} / \mathrm{s}$, even for a wide-bandwidth signal. These results confirm that a significant improvement is obtained for a SAC-OCDMA system by using optimized codes with low cross correlation, higher optical bandwidth, and optimized FBGs spectral responses with low guard band, as recommended in [10].

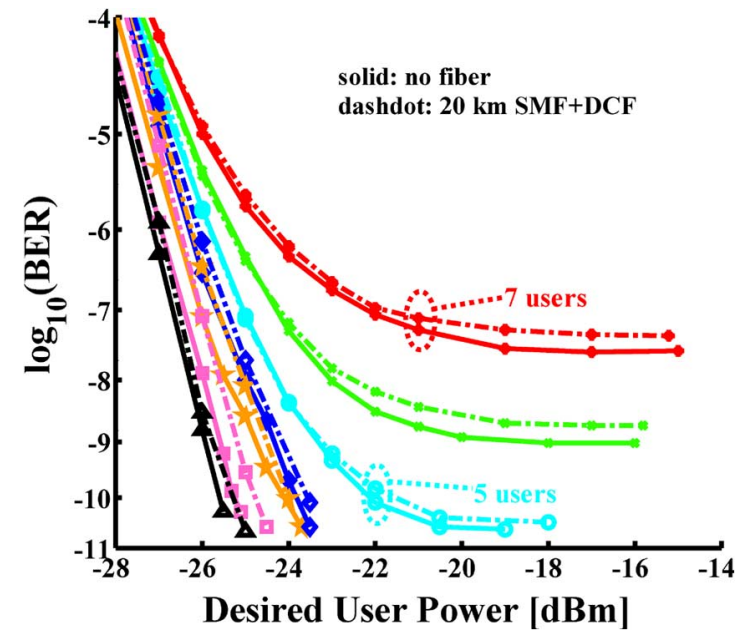

Fig. 9. BER versus power of the desired user without fiber and with $20 \mathrm{~km}$ of $\mathrm{SMF}+\mathrm{DCF}$.

We note that our experimental performance actually improved compared to that predicted via simulation (Fig. 6); in our analysis, we assumed that the signals from the two branches of the balanced photodetector were statistically independent, despite overlapping spectra. In fact, (7) and (8) are only valid if the spectra on the DEC and C-DEC arms are orthogonal. For the proposed spectral responses, this condition is not achieved as overlapping occurs. The common part of the spectral responses sends identical PSD to each photodiode. The intensity noise generated by that common part is canceled out by means of a perfectly matched balanced photodetector.

\section{CONCLUSION}

In this paper, we examined the optimization of FBG encoders for a SAC-OCDMA system. This optimization began by identifying the tradeoff between MAI and intensity noise when allowing overlap of realistic nonrectangular frequency bins. Overlapping can lead to excessively unequal QoS among users unless care is taken in the selection of the code family. We selected a code family with unity cross correlation and cyclic codes that gave roughly equal QoS among users. The degree of overlap of adjacent bins was varied via simulation, and a numerical optimization of the apodization profile was performed numerically to minimize the BER.

Once the optimal apodization was found, gratings were written for a code family whose length could be accommodated with phase masks that are readily available in our laboratory. We measured BER for a fully populated system (all seven codes in the code family) over a 9.6-nm optical bandwidth and a bit rate of $622 \mathrm{Mb} / \mathrm{s}$ for both back-to-back operation and 20-km single-mode fiber (SMF) with DCF. Error-free performance $\left(\right.$ BER $\left.<1 \times 10^{-9}\right)$ was achieved for six users back to back and five users over a $20 \mathrm{~km}$ fiber link. Error floors were in a range that could be easily lowered by the use of forward-error correction. Compared to previous SAC-OCDMA experimentation, we have achieved much improved performance (many orders of magnitude) and increased capacity with reduced cost encoders, i.e., chirped (not superimposed) FBG working in transmission. 
Future work will focus on increasing the number of users to be in the range of $16-32$ to match PON requirements and examination of low-cost components for that market.

\section{REFERENCES}

[1] A. Leon-Garcia, Communication Networks: Fundamental Concepts and Key Architectures, 2nd ed. New York: McGraw-Hill, May 2003.

[2] P. Prucnal, M. Santoro, and F. Ting, "Spread spectrum fiber-optic local area network using optical processing," J. Lightw. Technol., vol. LT-4, no. 5, pp. 547-554, May 1986.

[3] J. A. Salehi, A. M. Weiner, and J. P. Heritage, "Coherent ultrashort light pulse code-division multiple access communication systems," J. Lightw. Technol., vol. 8, no. 3, pp. 478-491, Mar. 1990.

[4] D. Zaccarin and M. Kavehrad, "An optical CDMA based on spectral encoding of LED," IEEE Commun. Lett., vol. 4, no. 4, pp. 479-482, Apr. 1993.

[5] G. A. Magel, G. D. Landry, R. J. Baca, D. A. Harper, and C. A. Spillers, "Transmission of eight channels $\times 622 \mathrm{Mb} / \mathrm{s}$ and 15 channels $\times 155 \mathrm{Mb} / \mathrm{s}$ using spectral encoded optical CDMA," Electron. Lett., vol. 37, no. 21, pp. 1307-1308, Oct. 2001.

[6] H. Fathallah, L. A. Rusch, and S. LaRochelle, "Passive optical fast frequency-hop CDMA communications system," J. Lightw. Technol., vol. 17, no. 3, pp. 397-405, Mar. 1999.

[7] R. P. Scott, W. Cong, C. Yang, V. J. Hernandez, N. K. Fontaine, J. P. Heritage, B. H. Kolner, and S. J. B. Yoo, "Error-free, 12-user, $10 \mathrm{~Gb} / \mathrm{s} / \mathrm{user}$ O-CDMA network testbed without FEC," Electron. Lett., vol. 41, no. 25, pp. 1392-1394, Dec. 2005.

[8] R. P. Scott, C. Wei, K. Li, V. J. Hernandez, B. H. Kolner, J. P. Heritage, and S. J. B. Yoo, "Demonstration of an error-free $4 / \mathrm{spl}$ times/ $10 \mathrm{~Gb} / \mathrm{s}$ multiuser SPECTS O-CDMA network testbed," IEEE Photon. Technol. Lett., vol. 16, no. 9, pp. 2186-2188, Sep. 2004.

[9] M. Abrams, P. C. Becker, Y. Fujimoto, V. O'Byrne, and D. Piehler, "FTTP deployments in the United States and Japan-Equipment choices and service provider imperatives," J. Lightw. Technol., vol. 23, no. 1, pp. 236246, Jan. 2005.

[10] S. Ayotte, M. Rochette, J. Magné, L. A. Rusch, and S. LaRochelle, "Experimental verification and capacity prediction of FE-OCDMA using superimposed FBG," J. Lightw. Technol., vol. 25, no. 2, pp. 724-731, Feb. 2005.

[11] J. W. Goodman, Statistical Optics. New York: Wiley, 2000.

[12] J. Penon, S. Ayotte, L. A. Rusch, and S. LaRochelle, "Incoherent SAC OCDMA system at $7 \times 622 \mathrm{Mbps,"} \mathrm{in} \mathrm{Proc.} \mathrm{CLEO,} \mathrm{Long} \mathrm{Beach,} \mathrm{CA,}$ May 2006, Paper CWH5.

[13] E. D. J. Smith, R. J. Blaikie, and D. P. Taylor, "Performance enhancement of spectral-amplitude-coding optical CDMA using pulse-position modulation," IEEE Trans. Commun., vol. 46, no. 9, pp. 1176-1185, Sep. 1998.

[14] E. D. J. Smith, P. T. Gough, and D. P. Taylor, "Noise limits of optical spectral-encoding CDMA systems," IEEE Electron. Lett., vol. 31, no. 17, pp. 1469-1470, Aug. 1995.

[15] J. Magné, D.-P. Wei, S. Ayotte, L. A. Rusch, and S. LaRochelle, "Experimental demonstration of frequency-encoded optical CDMA using superimposed fiber Bragg gratings," in Proc. Conf. BGPP, Monterey, CA, Sep. 2003, pp. 294-296.

[16] A. Papoulis and S. U. Pilai, Probability, Random Variables and Stochastic Processes, 4th ed. New York: McGraw-Hill, Jan. 2002, pp. 181-182.

[17] Z. Wei and H. Ghafouri-Shiraz, "Codes for spectral-amplitude-coding optical CDMA systems," J. Lightw. Technol., vol. 20, no. 8, pp. 12841291, Aug. 2002.

[18] J.-G. Zhang, "Design of nonconstant-weight strict optical orthogonal codes," Electron. Lett., vol. 41, no. 22, pp. 1238-1240, Oct. 2005.

[19] I. B. Djordjevic, B. Vasic, and J. Rorison, "Multi-weight unipolar codes for multimedia spectral-amplitude-coding optical CDMA systems," IEEE Commun. Lett., vol. 8, no. 4, pp. 259-261, Apr. 2004.

[20] S. A. Aljunid, M. Ismail, A. R. Ramli, B. M. Ali, and M. K. Abdullah, "A new family of optical code sequences for spectral-amplitude-coding optical CDMA systems," IEEE Photon. Technol. Lett., vol. 16, no. 10, pp. 2383-2385, Oct. 2004.

[21] Z. Wei, H. M. H. Shalaby, and H. G. Shiraz, "Modified quadratic congruence codes for fiber Bragg-grating-based spectral-amplitude-coding optical CDMA systems," J. Lightw. Technol., vol. 19, no. 9, pp. 12741281, Sep. 2001

[22] B. Honary, A. Moinian, and B. Ammar, "Construction of wellstructured quasi-cyclic low-density parity check codes," Proc. Inst. Electr. Eng.-Commun., vol. 152, no. 6, pp. 1081-1085, Dec. 2005
[23] J. Azaña and L. R. Chen, "Synthesis of temporal optical waveforms by fiber Bragg gratings: A new approach based on space-to-frequencyto-time mapping," J. Opt. Soc. Amer. B, Opt. Phys., vol. 19, no. 11, pp. 2758-2769, Nov. 2002.

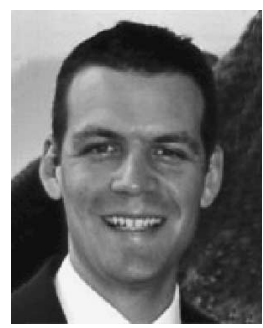

Julien Penon (S'06) was born in Amiens, France, in 1979. He received the Diplôme d'Etudes Approfondies degree in optics and photonics from Université Paris XI, Orsay, France, in 2003. He is currently working toward the Ph.D. degree in optical telecommunications with a dissertation on spectral-amplitude-coded optical code-division multiple access (OCDMA) at the Universite Laval, Sainte-Foy, QC, Canada.

His research interests include fiber-optic communication systems, fiber Bragg gratings, and OCDMA.

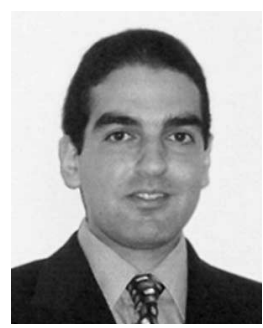

Ziad A. El-Sahn (S'98) was born in Alexandria, Egypt, in November 1979. He received the B.S and M.S. degrees in electrical engineering from Alexandria University, in 2002 and 2005, respectively. He is currently working toward the Ph.D. degree at the Université Laval, Sainte-Foy, QC, Canada.

In 2002, he joined the Department of Electrical Engineering, Alexandria University, where he was a Teaching Assistant for three years and was then promoted to Lecturer Assistant in 2005. His research interests include optical code-division multiple-access networks, passive optical networks, media-access-control protocols in optical networks, computer networks, and mobile communications.

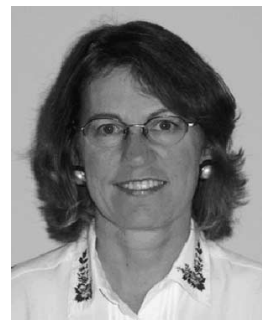

Leslie A. Rusch (S'91-M'94-SM'00) received the B.S.E.E. degree (with honors) from the California Institute of Technology, Pasadena, in 1980 and the M.A. and Ph.D. degrees in electrical engineering from Princeton University, Princeton, NJ, in 1992 and 1994, respectively.

She is currently with the Université Laval, SainteFoy, QC, Canada, where she is a Professor with the Department of Electrical and Computer Engineering and a member of the Center for Optics, Photonics, and Lasers. She spent two years (2001-2002) at Intel Corporation as the Manager of a group researching new wireless technologies. Her research interests include optical code-division multiple access (OCDMA) systems for passive optical networks, packet-switched networks with OCDMAbased labels, smart radio-over-fiber systems for spectrally efficient wireless local area networks, modeling of semiconductor optical amplifier dynamics for passive area networks and fiber-to-the-home, and optical processing for wireless UWB communications.

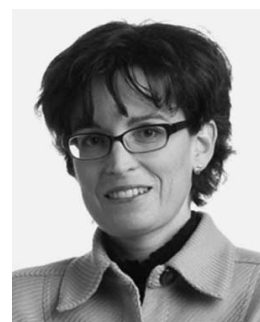

Sophie LaRochelle (M'00) received the B.Eng. degree in engineering physics from Université Laval, Sainte-Foy, QC, Canada, in 1987 and the Ph.D. degree in optics from the University of Arizona, Tucson, in 1992.

From 1992 to 1996, she was a Research Scientist at the Defense Research and Development CanadaValcartier, where she worked on electrooptical systems. She is currently a Professor at the Department of Electrical and Computer Engineering, Université Laval, where she holds a Canada Research Chair in Optical Fibre Communications and Components. She is a member of the Center for Optics, Photonics and Lasers. Her current research activities are focused on active and passive fiber optics components for optical communication systems, including fiber Bragg gratings, optical amplifiers, and multiwavelength and pulsed fiber lasers. Her other research interests include packet-switched networks with photonic code processing, transmission of radio-over-fiber signals, and optical code-division multiple access.

Dr. LaRochelle is a member of the Optical Society of America and the IEEE Lasers and Electro-Optics Society. 